\title{
Desalination Performance of Graphene Oxide-PEG Nanohybrid Membranes
}

\author{
Feng $\mathrm{ZHAO}^{1, \mathrm{a}}$,Fang $\mathrm{LI}^{1, \mathrm{~b}^{*}}$ and Chun-Yan $\mathrm{MA}^{1, \mathrm{c}}$ \\ ${ }^{1}$ College of environmental Science and Engineering, Donghua University, Shanghai 201620, China \\ a747220120@qq.com \\ blifang@dhu.edu.cn \\ 'machunyan@dhu.edu.cn
}

Keyword: GO, PEG, Crosslink, Salt rejection.

\begin{abstract}
In this work, the PEG-GO hybrid membranes fabricated by poly (ethylene glycol) diacrylate (PEGDA) and graphene oxide (GO) were tested for salt rejection. The prepared membranes with different proportions of PEG were characterized by morphology and chemical analysis as well as salt rejection experiments. The salt rejection experiments indicated the salt flux of the PEG-GO hybrid membranes reduce about $91 \%$ for $\mathrm{NaCl}, 75 \%$ for $\mathrm{CuSO}_{4}$ and $89 \%$ for $\mathrm{AlCl}_{3}$ respectively, exhibiting considerably superior to GO membrane. The sodium ions transfer more efficiently through the GO membranes than the heavy-metal salts.
\end{abstract}

\section{Introduction}

Nowadays, the development of membranes with novel separation functions is a key objective because of the scarcity of fresh water. The nano-materials played an important role in traditional membrane research. Current advances in material science led to the discovery of nanoparticles that have been used for many applications ranging from electronics, medical and even desalination applications. Nanocomposite membranes with the nano-scale channel provide water molecules with high speed transmission channel, and prevent macromolecules through it, thus realizing the desalination of sea water and desalination [1].

GO is gaining much more interest in the field of material research due to its high surface area, outstanding electron transport and mechanical properties. It has been confirmed that the mass transfer of mechanism high-molecular polymer of GO membrane is regarded as aperture screening model. In this work, PEG-GO nanohybrid membranes with GO nanosheets and PEG and were prepared to investigate the desalination performance [2].

\section{Materials and Methods}

\section{Materials}

PEGDA (MW=700), and 1-hydroxycyclohexyl phenyl ketone (HCPK) were purchased from Aldrich Chemical Company. Graphite powder was provided by the Qingdao Ruisheng Graphite Company. $\mathrm{H}_{2} \mathrm{SO}_{4}, \mathrm{KMnO}_{4}, \mathrm{H}_{2} \mathrm{O}_{2}(5 \%), \mathrm{HCl}, \mathrm{NaNO}_{3}$ of $\mathrm{AR}$ grade were purchased from Sinopharm Chemical Reagent Co. Ltd. 


\section{Membrane Fabrication}

GO was prepared by the modified Hummers' method using natural graphite as the source [3]. The PEG-GO nanohybrid membranes were fabricated by filter pression with cellulose acetate microfiltration membrane. The nanohybrid membranes were proportionally composed of PEG and GO. HCPK was added in the membrane casting solution as the initiator under the irradiation of UV light for two minutes.

\section{Characterization}

The atomic force microscopy (AFM) measurements were conducted with Nanoscope IV Multi Mode Controller (Veeco, USA) using the software supplied by the manufacturer. To characterize the functionalized polymers and membrane surfaces, ATR-FTIR Spectroscopy (Bruker, Tensor27, and German) and X-ray diffraction (Rigaku D/max-2500) were performed. Raman spectra were recorded on a Renishawin Via Reflex spectrometer using a $532 \mathrm{~nm}$ laser.

\section{Permeation Performance}

The desalination performances of GO and PEG-GO membranes were tested in filtration cell with effective membrane area of $0.0314 \mathrm{~cm}^{2}$. The salt flux $\mathrm{J}\left(\mathrm{g} /\left(\mathrm{m}^{2} \mathrm{~h}\right)\right)$ was calculated according to:

$$
J=\frac{\Delta C}{A \Delta T}
$$

Where, $\Delta \mathrm{C}$ was the concentration change of feed solution. A was the effective membrane area, and $\Delta \mathrm{T}$ was the filtration time.

\section{Results and Discussions}

\section{Membrane Characterization}
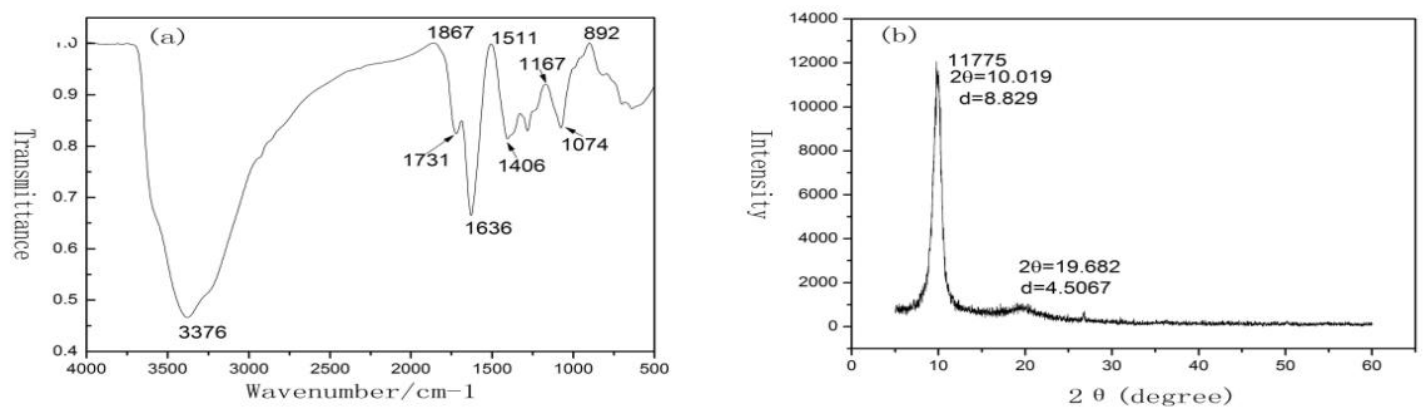

Fig.1 (a) FTIR spectra and (b) XRD pattern of GO nanosheets

In order to confirm the success of GO fabrication, the GO was characterized by the FTIR and XRD test. As shown in the Fig. 1(a), the presence of $\mathrm{O}-\mathrm{H}$ groups $\left(3376 \mathrm{~cm}^{-1}\right), \mathrm{C}=\mathrm{O}$ bonds in carboxyl groups $\left(1731 \mathrm{~cm}^{-1}\right)$, unoxidized $\mathrm{sp}^{2}$ aromatic $\mathrm{C}=\mathrm{C}$ bonds $\left(1636 \mathrm{~cm}^{-1}\right)$, and $\mathrm{C}-\mathrm{O}$ bonds in epoxy groups $\left(1074 \mathrm{~cm}^{-1}\right)$ confirmed the synthesized GO [4]. According to the XRD patterns, the sharp peak at $2 \theta=10.0^{\circ}$ and other peak at $2 \theta=19.7^{\circ}$ were corresponded to the characteristic reflection of GO [5]. 

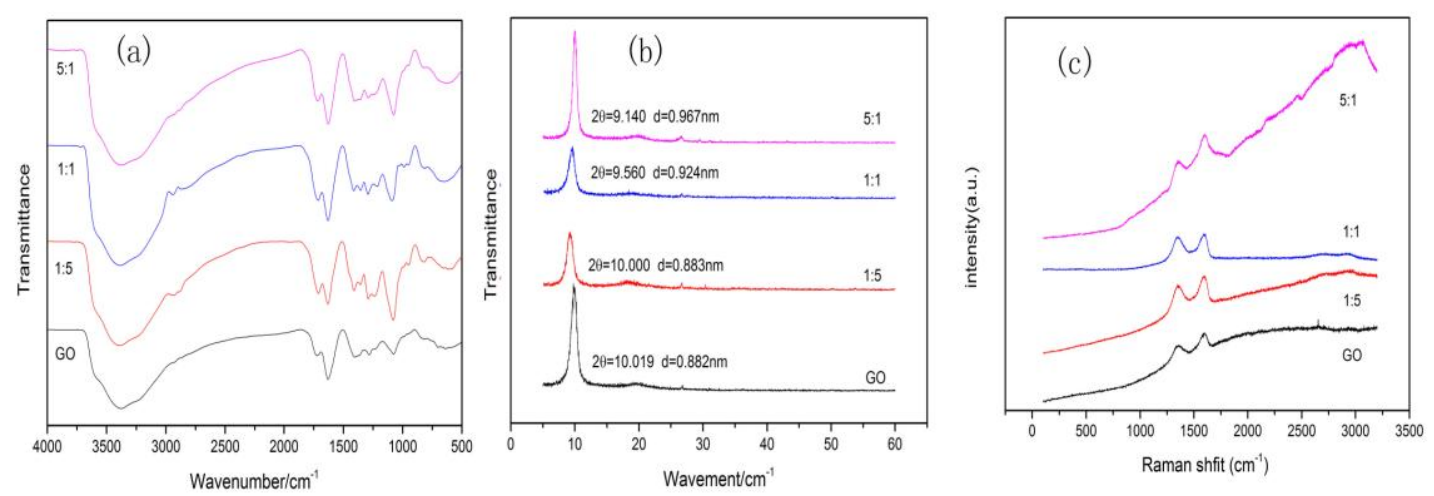

Fig.2 (a) FTIR spectra (b) XRD pattern (c) Raman spectra of GO and PEG-GO hybrid membrane

As shown by the FTIR spectra in Fig. 2(a), there was a new small peak appeared in $1380 \mathrm{~cm}^{-1}$, which indicated that GO had been cross linked by PEG. The reason was that hydroxyl and carboxyl possibly reacted and produced ester bond in acid solution. The structure evolution of GO and PEG-GO hybrid membrane were studied by XRD, as shown in Fig. 2(b). Stacked GO nanosheets showed typical broad diffraction peak at $2 \theta=10.0^{\circ}$ while pure PEG membranes showed a sharp peaks at $2 \theta=21.4^{\circ}$. It can be found that the $2 \theta$ was reduced with the increase of the proportion of PEG. This shifting revealed the successful intercalation of PEG into the interlayer of GO nanosheets. The PEG-GO hybrid membrane showed a sharp at $2 \theta=9.1^{\circ}$. The XRD spectra (Fig. 2(b)) also showed the change of the interlayer distance (d-spacing). Obviously, interlayer distance (d-spacing) increased from $0.882 \mathrm{~nm}$ for GO membrane to $0.967 \mathrm{~nm}$ for PEG-GO hybrid membrane (PEG: GO=5:1). Raman spectra of GO and PEG-GO hybrid membranes, explained $\mathrm{G}$ band at 1576 $\mathrm{cm}^{-1}$ and $\mathrm{D}$ band at $1348 \mathrm{~cm}^{-1}$, respectively, which were attributed to the crystallinity of $\mathrm{sp}^{2}$ hybrid carbon regions and the carbon lattice distortion [6]. The enlarged intercalated channels imparted more structural disorder into the GO laminate structures. The results also revealed that the defects of GO nanosheets were bigger and the disorder degree of membrane increased, which was constant with the result of XRD.

The roughness of the GO and PEG-GO hybrid membranes were characterized by AFM and the results were summarized in Table 1 . The roughness of the membrane declined with the loading of PEG until the ratio of GO: PEG reached 1:1.

Table1. Surface roughness of the membranes by AFM

\begin{tabular}{lll}
\hline Membrane & $\mathrm{R}_{\mathrm{a}} \mathrm{nm}$ & $\mathrm{R}_{\mathrm{q}} \mathrm{nm}$ \\
\hline GO & 81.0 & 97.3 \\
GO:PEGDA=1:5 & 67.6 & 80.2 \\
GO:PEGDA=1:1 & 43.7 & 55.4 \\
GO:PEGDA=5:1 & 75.1 & 92.7 \\
\hline
\end{tabular}




\section{Ion-Penetration}
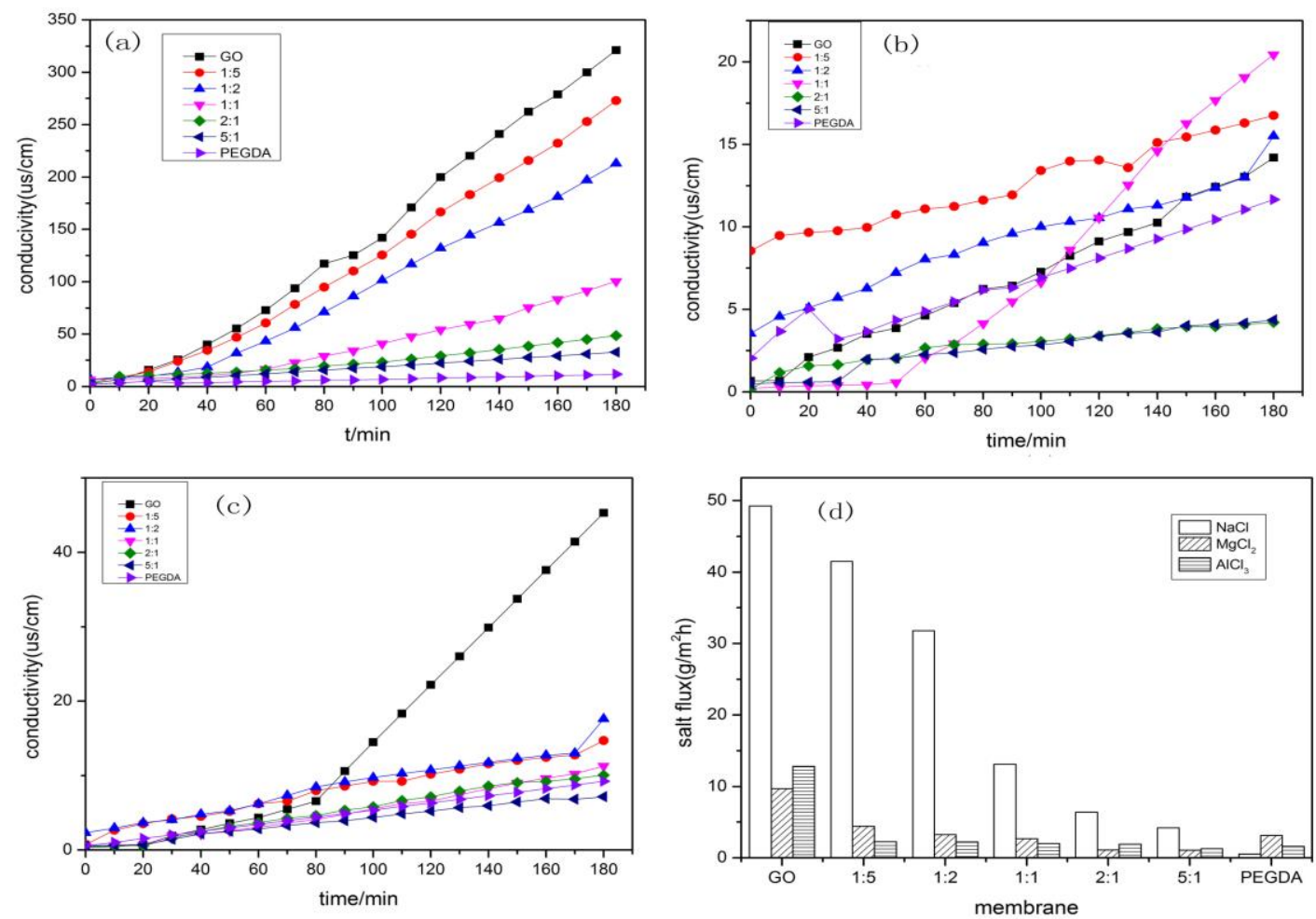

Fig.3 Ion-penetration experiments of (a) $\mathrm{NaCl}$ (b) $\mathrm{CuSO}_{4}$ (c) $\mathrm{AlCl}_{3}$ (d) salt flux

A series of ion-penetration experiments were conducted with salt solution of $0.1 \mathrm{~mol} / \mathrm{L}$ solution of $\mathrm{NaCl}, \mathrm{AlCl}_{3}$ and $\mathrm{CuSO}_{4}$, as shown in the Fig. 3. The results of the ion-penetration were presented in the change of the conductivities of the feed solution [7]. As was illustrated in Fig. 3, the conductivity profiles of three solutions exhibited similar tendencies. For the $\mathrm{NaCl}$ salt solutions, with the increase of the proportion of PEG, the change of salt permeability was less significant than the other solutions. The salt flux of PEG-GO membrane permeated with the solution of $\mathrm{NaCl}$, $\mathrm{CuSO}_{4}$ and $\mathrm{AlCl}_{3}$ were 49.25, 4.41, and $12.79 \mathrm{~g} /\left(\mathrm{m}^{2} \mathrm{~h}\right)$ while the salt flux of PEG-GO hybrid membrane (PEG:GO=5:1) were 4.21, 1.08 , and $1.31 \mathrm{~g} /\left(\mathrm{m}^{2} \mathrm{~h}\right)$, respectively. The penetration property of GO membrane was originated from the fact that thousands of GO nanosheets were stacked into a lamellar structure forming GO membrane. As for the PEG-GO nanohybrid membranes, the GO nanosheets were crosslinked with high-molecular polymer, i.e. PEG. With the increment loading of PEG, the dimensional nanochannel between the GO nanosheets was filled by the PEG moleculars. Therefore, the nanohybrid membranes presented a more excellent salt rejection than GO membranes. On the other hand, the mass transfer mechanism of the nanohybrid membranes changed with the PEG loading. As shown in the result, the mass transfer mechanism of the GO-based membranes was dominated by size-sieve principle while the PEG organic membranes were dissolving-diffusion model. As the substance proportion in the nanohybrid membranes changed, the mass transfer mechanism was changed correspondingly. The permeability of different salt solutions changed dramatically with the ion size. The sodium ions transfer efficiently through the GO membrane whereas the heavy-metal salts transfer slowly. As it was well-known, the hydrated diameter of $\mathrm{Na}^{+}$was $0.72 \mathrm{~nm}$ and the average interlayer spacing of GO nanosheets was $0.88 \mathrm{~nm}$ as obtained by XRD tests. Consequently, $\mathrm{Na}^{+}$could pass more fast through the GO membranes than $\mathrm{Cu}^{2+}$ and $\mathrm{Al}^{3+}$. 


\section{Conclusions}

In the nanohybrid membranes, the PEG moleculars played a role as a crosslinker to form the GO nanosheets to into stable stacked membranes. With the increase of the PEG proportions in the nanohybrid membrane, the desalination performance of PEG-GO hybrid membranes were better. The salt rejection experiments indicated the salt flux of the PEG-GO hybrid membranes reduce about $91 \%, 75 \%$ and $89 \%$, corresponding to $\mathrm{NaCl}, \mathrm{CuSO}_{4}$ and $\mathrm{AlCl}_{3}$, respectively, considerably higher than that of GO membranes. In brief, it can be concluded that the PEG-GO hybrid membranes are superior to GO membrane in desalination.

\section{Acknowledgements}

This work is supported by the National Natural Science Foundation of China (51478099, 51508082), national key research and development plan (2016YFC0400503) and the Fundamental Research Funds for the central University (223215A3-04).

\section{Reference}

[1] Xia SJ, Yao LJ, Zhao Y, Li NN, Zheng Y. Preparation of graphene oxide modified polyamide thin film composite membranes with improved hydrophilicity for natural organic matter removal. Chemical Engineering Journal. 2015, 280:720-7.

[2] Smith ZP, Freeman BD. Graphene Oxide: A New Platform for High-Performance Gas- and Liquid-Separation Membranes [J]. Angew Chem-Int Edit. 2014, 53(39):10286-8.

[3] Marcano DC, Kosynkin DV, Berlin JM, Sinitskii A, Sun ZZ, Slesarev A, et al. Improved Synthesis of Graphene Oxide[J]. Acs Nano. 2010,4(8):4806-14.

[4] Liang Y, Yatao Z, Bing Z, Jindun L, Haoqin Z, Chunhua S. Preparation and characterization of HPEI-GO/PES ultrafiltration membrane with antifouling and antibacterial properties[J]. Journal of Membrane Science. 2013,447:452-62.

[5] Huiqing W, Beibei T, Peiyi W. Development of novel SiO2-GO nanohybrid/polysulfone membrane with enhanced performance [J]. Journal of Membrane Science. 2014,451:94-102.

[6] Zhao XT, Su YL, Liu YA, Lip YF, Jiang ZY. Free-Standing Graphene Oxide-Palygorskite Nanohybrid Membrane for Oil/Water Separation [J]. Acs Applied Materials \& Interfaces. 2016, 8(12):8247-56.

[7] Sun P, Ma R, Deng H, Song Z, Zhen Z, Wang K, et al. Intrinsic high water/ion selectivity of graphene oxide lamellar membranes in concentration gradient-driven diffusion [J]. Chemical Science. 2016, 7(12):6988-94. 